\title{
Perspective
}

PERSPECTIVE Actualité en histoire de l'art

$1 \mid 2020$

Japon

\section{Conceptions de l'ornement en quête de discours scientifique}

Ornament Designs in Search of a Scientific Foundation

Ornamentale Entwürfe auf der Suche nach einem wissenschaftlichen Diskurs

Concezioni dell'ornamento alla ricerca di un discorso scientifico

Concepciones del ornamento en búsqueda de discurso científico

\section{Caroline van Eck}

Traducteur : François Boisivon

\section{(2) OpenEdition}

\section{Journals}

Édition électronique

URL : http://journals.openedition.org/perspective/19667

DOI : 10.4000/perspective.19667

ISSN : 2269-7721

Éditeur

Institut national d'histoire de l'art

\section{Édition imprimée}

Date de publication : 5 juin 2020

Pagination : 353-362

ISBN : 978-2-917902-89-9

ISSN : $1777-7852$

Référence électronique

Caroline van Eck, "Conceptions de l'ornement en quête de discours scientifique », Perspective [En ligne], 1 | 2020, mis en ligne le 30 décembre 2020, consulté le 25 janvier 2021. URL : http://

journals.openedition.org/perspective/19667; DOI : https://doi.org/10.4000/perspective.19667 


\title{
Conceptions de l'ornement en quête de discours scientifique
}

\author{
Caroline van Eck
}

\author{
- GUEST, 2013 : Claire Guest, The Understanding of Ornament in the \\ Italian Renaissance, Leyde / Boston, Brill, 2013. \\ - LABRUSSE, 2018 : Rémi Labrusse, Face au chaos. Pensées de l'ornement \\ à l'âge de l'industrie, Dijon, Les Presses du réel, 2018. \\ - NECIPOĞLU et PAYNE, 2016 : Gülru Necipoğlu et Alina Payne (dir.), \\ Histories of Ornament. From Global to Local, Princeton (N.-J.), Princeton \\ University Press, 2016.
}

Les tentatives d'écrire une histoire occidentale de l'ornement sont rares. Elles s'enlisent souvent, les créateurs identifiés étant trop peu nombreux, les sources et les travaux théoriques trop clairsemés, mais aussi pour des raisons plus profondes de méthode. Les histoires existantes s'attachent le plus souvent aux développements formels d'un support, d'un type spécifique d'objets ou d'une discipline artistique, par exemple le mobilier, les pièces de cheminées ou encore l'ornement dans l'architecture gothique. Il n'existe pas d'histoires d'objets décoratifs aussi anciens et répandus que le candélabre ou le tripode qui embrasseraient une période tant soit peu étendue, et l'on peut faire le même constat pour des motifs ornementaux comme la feuille d'acanthe. Un autre facteur complique encore les choses : bien souvent, l'ornement n'est pas exclusivement lié à un médium, à une discipline, à un matériau ou à un genre : la feuille d'acanthe migre de la poterie à l'architecture et à la sculpture, du bois à la céramique ou au marbre, des temples consacrés à Apollon aux boudoirs rococo ${ }^{1}$. En conséquence de cette mobilité, de cette propension à la métamorphose, l'ornement cesse d'être une question de portée théorique ou historique générale, le cédant à la spécificité du matériau ou de la période dans le champ esthétique ou dans celui de la théorie des arts, comme en témoignèrent, par exemple, les débats suscités par le Laocoon de Lessing sur l'unicité des arts visuels. C'est à l'architecte dresdois Friedrich August Krubsacius (1718-1789) que l'on doit la première tentative de rédaction d'une histoire de l'ornement en Europe, mais elle est encore essentiellement fondée sur les récits mythiques et les spéculations de Vitruve à propos des origines de l'ornement, repris par Alberti ${ }^{2}$. Ce n'est qu'à partir du moment où l'on fait venir des objets du monde entier, à la fin du XVIII ${ }^{e}$ siècle, que l'on commence à rédiger des histoires de l'ornement qui s'écartent des paramètres de la rhétorique classique et des théories de Vitruve, ayant jusqu'alors constitué les deux principales sources occidentales sur le sujet. 
La distinction introduite dans les traités de rhétorique d'Aristote, de Cicéron et plus particulièrement de Quintilien, entre la matière de ce que l'on dit, fait ou fabrique et la manière dont on le dit, fait ou fabrique marque longtemps la plupart des théories occidentales de l'ornement. Dans les manuels de rhétorique, elle correspond à la distinction entre res, l' " objet " et la " substance " du discours, et verba, les " mots " utilisés pour présenter cette substance de la manière la plus persuasive. Elle n'est pertinente que lorsque la formulation résulte d'un choix. Dans la rhétorique classique, ce choix dépend étroitement de la situation, du sujet traité, de l'auditoire ; et l'orateur, en visant la plus grande force de persuasion, devait respecter le decorum. C'est précisément cette notion de choix qui fut également déterminante dans la plupart des considérations occidentales sur l'ornement, lequel était presque toujours associé à l'apparence extérieure, à la décoration en surface, et non à la substance ou à l'essence du discours ou de tout autre objet culturel. Quoique originellement créée comme une théorie du discours de la persuasion, la rhétorique touchait tout ce qui avait trait à la communication entre les humains, qu'elle ait lieu par la parole, le geste ou l'image. Ainsi les grands traités classiques, ceux d'Aristote, de Cicéron, de Quintilien et de Longin traitent tous de l'emploi des arts visuels pour convaincre un auditoire. Composé comme un manuel à l'attention des jeunes orateurs, L'Institution oratoire (De institutione oratoria) de Quintilien offre une synthèse pratique et théorique, grecque et romaine, de la rhétorique, dont la mode n'est jamais passée. Des exemples visuels éloquents sont appelés en renfort pour illustrer les figures du discours, pour le rendre plus captivant, partant, plus persuasif ${ }^{3}$. La figure du Discobole de Myron, par exemple, convoquée pour illustrer les effets de l'antithèse aura une belle postérité dans les débats de la Renaissance sur le contrapposto ${ }^{4}$. Parallèlement, Quintilien introduit une autre relation déterminante : entre ornement et style, en se livrant à une première tentative de classification de la sculpture fondée sur des traits stylistiques, eux-mêmes dérivés des définitions rhétoriques des styles oratoires attribués aux écoles locales (le style attique était plus sobre, le style rhodien plus fleuri et recherché), qui se distinguent par les figures du discours - et de la pensée - et, d'une façon générale, par les éléments relevant de leur ornementation ou ornatus.

L'autre grande source de la pensée occidentale de l'ornement est la théorie grécoromaine de l'architecture, du moins ce qui en a survécu dans les dix livres du De architectura de Vitruve. Imbu des notions rhétoriques de decorum et d'ornatus, Vitruve considère que les ordres architecturaux et l'entablement qu'ils soutiennent constituent l'élément ornemental principal de l'architecture. Ils inspirent aussi la plupart des composants ornementaux utilisés sur l'extérieur des bâtiments. Lorsqu'il traite des ordres, le mot qu'emploie Vitruve est ornamentum, mais dans le sens d'une représentation dans la pierre des constructions primitives en bois, non dans celui d'un élément ajouté. Leur usage est codifié par la coutume, la tradition et le decorum ou convenance 5 . Si Vitruve condamnait les peintures murales du second style pompéien, avec leurs représentations élaborées de candélabres, de masques de théâtre et de portiques, mêlés sans aucun souci de logique structurelle, c'est qu'il pensait que l'art ne devait dépeindre que ce qui existe, en obéissant aux lois de la nature. Les termes de sa réfutation ont eu une longue postérité : Vasari, par exemple, les utilise, à la Renaissance, pour décrier le gothique, tout comme les néoclassiques du XVIII ${ }^{\mathrm{e}}$ siècle les reprendront pour condamner l'irrégularité des formes du rococo et leur refus des iconographies claires et conventionnelles comme le symbolisme héraldique $^{6}$. Ainsi le cadre conceptuel principal qui permet de théoriser l'ornement en Occident s'organise-t-il suivant deux axes : l'un est rhétorique et se fonde sur la distinction entre la matière et la manière du propos; l'autre est architectural et tourne autour de l'opposition entre structure - ou fonction - et apparence. On les trouve souvent mêlés, comme en témoigne le choix que fait Alberti dans De re aedificatoria de traiter deux fois 
des ordres, une première fois comme élément structurel des bâtiments, une seconde fois comme leur ornement principal, dont l'usage est dicté par des considérations rhétoriques de persuasion et de convenance.

Ce cadre conceptuel se brise pour une bonne part au XIX ${ }^{\mathrm{e}}$ siècle. Le champ géographique et chronologique des études sur l'ornement s'étend considérablement, ce qui contribue à la naissance d'une nouvelle façon, anthropologique, de le penser. L'arrivée en Europe d'objets en provenance du Pacifique, dans les années 1760, suscite les premières tentatives de mettre en place une perspective globale, ethnographique. Ainsi l'archéologue Carl August Böttiger propose-t-il le tatouage comme l'une des variétés princeps de l'ornement, suggestion que retiendra Gottfried Semper 7 . En 1856, Owen Jones publie sa Grammaire de l'ornement ${ }^{8}$, première tentative sérieuse d'établir une morphologie globale des motifs ornementaux. Changeant fondamentalement de paradigme, Jones abandonne le cadre rhétorique et architectural, pour lui substituer un modèle linguistique. S'inspirant de la discipline émergente de la linguistique comparative, il isole des motifs originaux et examine leurs transformations dans le temps et dans l'espace pour structurer l'évolution de l'ornement.

Le modèle linguistique fut aussi une source importante d'inspiration pour Semper. À la différence de Jones et de son modèle linguistique pourtant, il considère que les quatre activités premières de la fabrique humaine des artefacts (le tissage, la charpente, la maçonnerie et la métallurgie) fournissent les éléments à partir desquels tout ornement s'est développé ; il passe ainsi du paradigme linguistique à un paradigme anthropologique, où l'activité humaine, et non le langage, devient l'origine de l'ornement, comme de toute culture matérielle. La représentation au cours du temps et sur différents médiums de ces arts manuels, par exemple celle de structures tissées dans la décoration en terre cuite ou bien celle des bordures d'une tapisserie sur un élément d'encadrement, constitue le fondement même de l'ornement dans les cultures humaines depuis la préhistoire jusqu'aux temps présents.

La première Exposition universelle, qui se tint à Londres, en 1851, élargit non seulement l'horizon de l'étude de l'ornement au monde entier, mais elle est aussi le catalyseur d'une réflexion systématique sur les arts décoratifs et sur la façon dont la production de masse influence le savoir-faire et la conception des objets d'usage. L'ouvrage de Gottfried Semper, Science, industrie et art ${ }^{9}$, publié en 1852, fut l'une des premières tentatives d'analyser les relations entre l'architecture et les arts appliqués et d'en tirer les conséquences pour les formations aux arts décoratifs. Dans son livre qui aura le plus d'influence, Le Style dans les arts techniques et tectoniques, ou Esthétique pratique... ${ }^{10}$ (1860-1863), Semper retrace l'évolution des quatre artisanats fondamentaux, partant de leurs premières manifestations dans la Caraïbe et le Pacifique, passant par leurs transformations dans les textiles, les céramiques et les constructions en brique des Égyptiens, des Assyriens et des Romains, jusqu'au début de la période moderne. Considérant que se masquer et se vêtir - c'est-à-dire décorer des surfaces - sont les opérations essentielles de l'art, il transforme radicalement le statut de l'ornement. En opposition à l'appréciation qu'en donne Kant qui le réduit à la manifestation d'une beauté purement formelle et gratuite, Semper ramène l'ornement au cœur même de l'évolution artistique de l'humanité.

\section{Lustre originel de l'ornement}

Les trois livres dont il est ici rendu compte jettent tous une lumière nouvelle sur cette histoire complexe. The Understanding of Ornament in the Italian Renaissance, de Claire Guest, offre beaucoup plus que ne le suggère son titre. Bien mieux qu'une simple synthèse de la théorie de l'ornement à la Renaissance, c'est une tentative extrêmement ambitieuse et érudite 


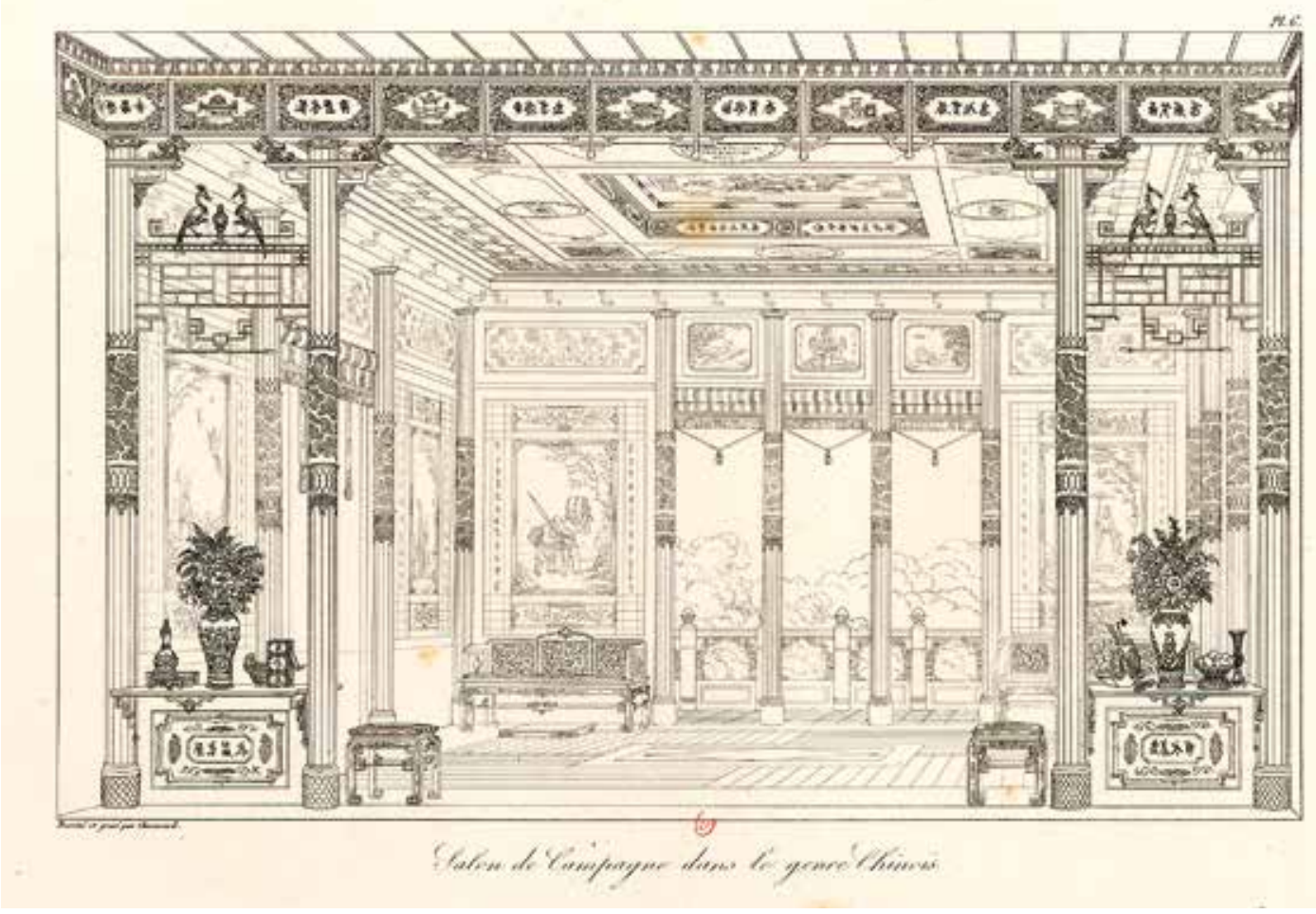

1. Claude-Aimé Chenavard, «Salon de campagne dans le genre chinois ", pl. 6 du Recueil des dessins de tapis, tapisseries et autres objets d'ameublement exécutés dans la manufacture de $M$. Chenavard, Paris, Émile Leconte, vers 1833. Paris, bibliothèque de l'Institut national d'histoire de l'art, collections Jacques Doucet, NUM FOL EST 616. de retrouver la pré-histoire des concepts liés à l'ornement tels qu'ils ont évolué depuis la fin du XVIII ${ }^{\mathrm{e}}$ siècle, après l'émergence de l'esthétique dans la philosophie, marquée notamment par la conception kantienne qui fait des jugements esthétiques l'expression du libre jeu des facultés cognitives et perceptuelles et par conséquent de l'ornement le domaine d'une beauté libre, sans contrainte, mais par là même sans but. Guest remonte aux origines de la pensée occidentale de l'ornement, dans l'Ancien Testament, dans la pensée grecque platonicienne, aristotélicienne ou sophistique, et jusque dans la scholastique, pour en extraire une conception de la beauté et de l'ornement qui ne soit pas déterminée à partir d'un concept relatif de la beauté qui conçoit cette dernière comme une sensation émergeant dans la perception d'un objet par ses spectateurs, mais résonne avec l'idée grecque, reprise au Moyen Âge, selon laquelle l'ornement serait l'éclat (splendor) de la vérité et de la beauté. "L'ornement - expose l'auteur en introduction n'était pas seulement une chose, mais une manière de percevoir et de concevoir " ; il était conçu comme intrinsèque, non pas relationnel, et également pertinent pour les humains, les objets et les comportements.

Au cours de cet exercice érudit, Guest revisite les conceptions de la Renaissance sur l'ornement, notamment celles d'Alberti, réexamine le grotesque, le triomphe et le fragment, et affirme que dès la Renaissance, en prenant le Courtisan de Castiglione comme point de basculement, les fondements du concept esthétique de l'ornement étaient jetés. Le livre se distingue par sa connaissance philologique et sa portée philosophique. Il enrichit substantiellement les conceptions traditionnelles de l'histoire de l'art selon lesquelles la 
pensée de l'ornement à la Renaissance dérive essentiellement de la théorie architecturale. Cependant, cet exercice d'archéologie esthétique laisse ouvert, en son cœur même, un étrange anachronisme : les opinions communément admises sur l'histoire de la pensée de l'ornement et de la beauté à la Renaissance sont passées au crible et révisées à la lumière d'une abondance sans précédent de sources, mais les conceptions de l'esthétique kantienne et leur impact sur la théorie de l'ornement, analysé par Gadamer et Derrida, ne sont pas elles-mêmes interrogées, pas plus que l' « historicisme " élaboré que Guest attribue à la pensée de la Renaissance. Ainsi ce projet de relecture et de remise en question évite dans une grande mesure la question de l'esthétique kantienne et de ses conséquences sur le XIX ${ }^{e}$ siècle. Quoi qu'il en soit, toute étude sérieuse de la pensée classique, médiévale ou renaissante sur l'ornement devra tenir compte des arguments développés par Guest.

\section{Changements de paradigmes}

Le livre de Rémi Labrusse est une étude aussi dense que subtile sur les tentatives de repenser la question de l'ornement au XIX ${ }^{e}$ siècle, de sa conception et de son histoire, face à tous les enjeux évoqués plus haut : l'émergence d'une perception de l'art et de la fabrique d'ornements comme des phénomènes de nature globale, et les changements radicaux imposés par la révolution industrielle à la production artistique. C'est un point de départ radicalement nouveau comparé aux études existantes sur l'ornement au XIX ${ }^{\mathrm{e}}$ siècle et sur les principaux théoriciens qui s'y intéressèrent alors - Jones, Semper, Bötticher, Charles Blanc -, parce que Labrusse ne s'attache ni aux formes et à leur réalisation, ni à l'impact des théoriciens mentionnés sur la production artistique, encore moins à la question de la collection et des conditions de présentation de l'ornement et des arts manuels, mais aux fondements intellectuels des théories de ces auteurs. La trajectoire empruntée par le livre est double : méthodologique, car Labrusse renonce à l'historicisme et à l'éclectisme comme principes directeurs d'un ordonnancement de l'histoire des théories du XIX $\mathrm{X}^{\mathrm{e}}$ siècle sur l'ornement pour tenter plutôt de dévoiler les paradigmes - parfois seulement les métaphores - employés par les auteurs eux-mêmes. La linguistique, et plus particulièrement le concept de grammaire, leur fournit le principal modèle, qui détermine à son tour l'autre trajectoire dessinée par l'ouvrage : la création de grammaires de l'ornement et les évolutions profondes de ce concept. La grammaire, qui avait commencé d'acquérir son prestige dans la société française du XVII siècle, apparut comme une métaphore pertinente pour classer toutes les sortes d'ornements dans les répertoires de motifs. Comme l'affirme Labrusse, elle devint un puissant antidote à la profusion des styles d'ornements présentés dans un cadre historiciste par un Claude-Aimé Chenavard, par exemple, dans son Album de l'ornemaniste, recueil composé de fragmens d'ornemens dans tous les genres et tous les styles, publié en 1836, ou dans son Recueil des dessins de tapis, tapisseries et autres objets d'ameublement..., publié vers 1833 (fig. 1).

De métaphore heuristique, le concept de grammaire s'est transformé, à mesure qu'avançait le siècle, en paradigme scientifique, s'appuyant sur les progrès de la linguistique comparative, où s'illustraient Wilhelm von Humboldt, l'helléniste Karl Otfried Müller ou Franz Bopp, le fondateur de la grammaire comparative des langues indo-européennes. Leurs travaux constituèrent un cadre qui permit de comprendre l'évolution et la variété de ces langues en employant des modèles qui rendaient compte des transformations des éléments originels jusqu'au sein des langues pleinement constituées. La grammaire devient ici le modèle grâce auquel on découvre les lois souterraines de l'évolution linguistique. Mais ces travaux permirent aussi de démontrer que les langues gréco-romaines, tout comme la plupart des langues européennes, appartenaient à une famille beaucoup plus 
vaste s'étendant de l'Islande à l'Inde. Il en découlait, pour Jones, par exemple, qu'il devait être possible de démontrer que les ornements des cultures humaines, comme les énoncés de celles-ci, obéissaient à un même principe grammatical. Mais ils devaient aussi, comme les langues, être étudiés dans un contexte global. On trouve ici, ainsi que le montre Labrusse dans l'une de ses nombreuses et perspicaces analyses, un étrange décalage. Si l'Orient des linguistes était d'abord hindou, les théoriciens de l'ornement concentrèrent leur attention sur l'ornement islamique, et plus particulièrement persan, parce que pour eux les Perses étaient, à l'instar des Européens, des Aryens, et les précepteurs du monde islamique, que les Arabes auraient imités.

Tout cela est déjà extrêmement instructif, mais la part la plus novatrice du livre de Labrusse réside dans le chapitre fondamental qu'il consacre à Bötticher et, dans une moindre mesure, à Semper. Si ce dernier a été partiellement traduit en français - et Isabelle Kalinowski prépare une édition critique complète de Der Stil -, Bötticher est à peu près inconnu en France, quoique la notion de " tectonique ", reprise par les architectes modernistes et, dans les années 1980 et 1990, par le théoricien Kenneth Frampton, ait entretenu sa postérité dans le monde anglo-saxon. Mais le chapitre de Labrusse va beaucoup plus loin, parce qu'il se livre à la première reconstruction en profondeur des idées de Bötticher et de leur contexte intellectuel. Et force est d'admettre que cet auteur compte, malgré l'aridité de son œuvre et même si ses idées en archéologie se sont avérées fausses, car il a totalement repensé la nature de l'ornement. Il a abandonné le paradigme grammatical et s'est plutôt inspiré des considérations esthétiques de l'idéalisme allemand selon lesquelles l'art est d'abord une représentation et la thématisation de cette représentation, en y adjoignant les théories linguistiques de Wilhelm von Humboldt qui concevait la formation des mots comme une concentration d'énergie, fondée sur les images mentales que forme le locuteur avant leur énonciation. Les deux grands concepts de Bötticher sont celui de Kunstformen ( "formes artistiques ") et celui de Werkformen ("formes opératoires ") : les premières sont l'apparence ou la manifestation visuelle des forces tectoniques - poids, structures portantes - à l'œuvre mais invisibles dans un bâtiment. S'appuyant sur la compréhension des forces, dans les sciences physiques, comme des abstractions plutôt que des phénomènes concrets, Bötticher affirme, dans une formule célèbre, que sans formes artistiques, un bâtiment serait muet et "immobile "; c'est seulement à travers ses formes artistiques, autrement dit l'ornement, qu'il parvient à parler, c'est-à-dire qu'il devient compréhensible à ceux qui le voient : "Muettes et figées, la pensée et les idées se trahissent seulement par des signes pleins de caractère ${ }^{11}$."

Recenser l'ensemble des enseignements de cette lecture approfondie de Bötticher et de leurs implications nous entraînerait bien au-delà des limites de ce compte rendu, mais un point doit être souligné, eu égard à son importance pour les tentatives qui suivront d'établir des histoires globales de l'ornement : en dépassant les paradigmes rhétorique, vitruvien ou grammatical traditionnels, et en élaborant à leur place une théorie de l'ornement qui est à la fois ontologique et herméneutique, dans laquelle il distingue les degrés de réalité des formes artistiques et des formes opératoires en fonction de leur manifestation dans le monde visible, tandis que son objet devient le fil même qui dirige sa pensée, Bötticher permet la mutation d'une théorie de l'ornement principalement esthétique et artistique vers une théorie anthropologique.

Semper complète cette mutation, d'une façon tout aussi radicale, quoique différente. Tandis que pour Bötticher l'architecture gréco-romaine était tout à la fois la source d'inspiration et le terrain d'expérimentation ultimes, et l'ornement dérivé des ordres antiques son domaine principal de recherche, Semper s'écarte résolument de la primauté de la tradition classique. Chez lui, les quatre activités artistiques humaines 


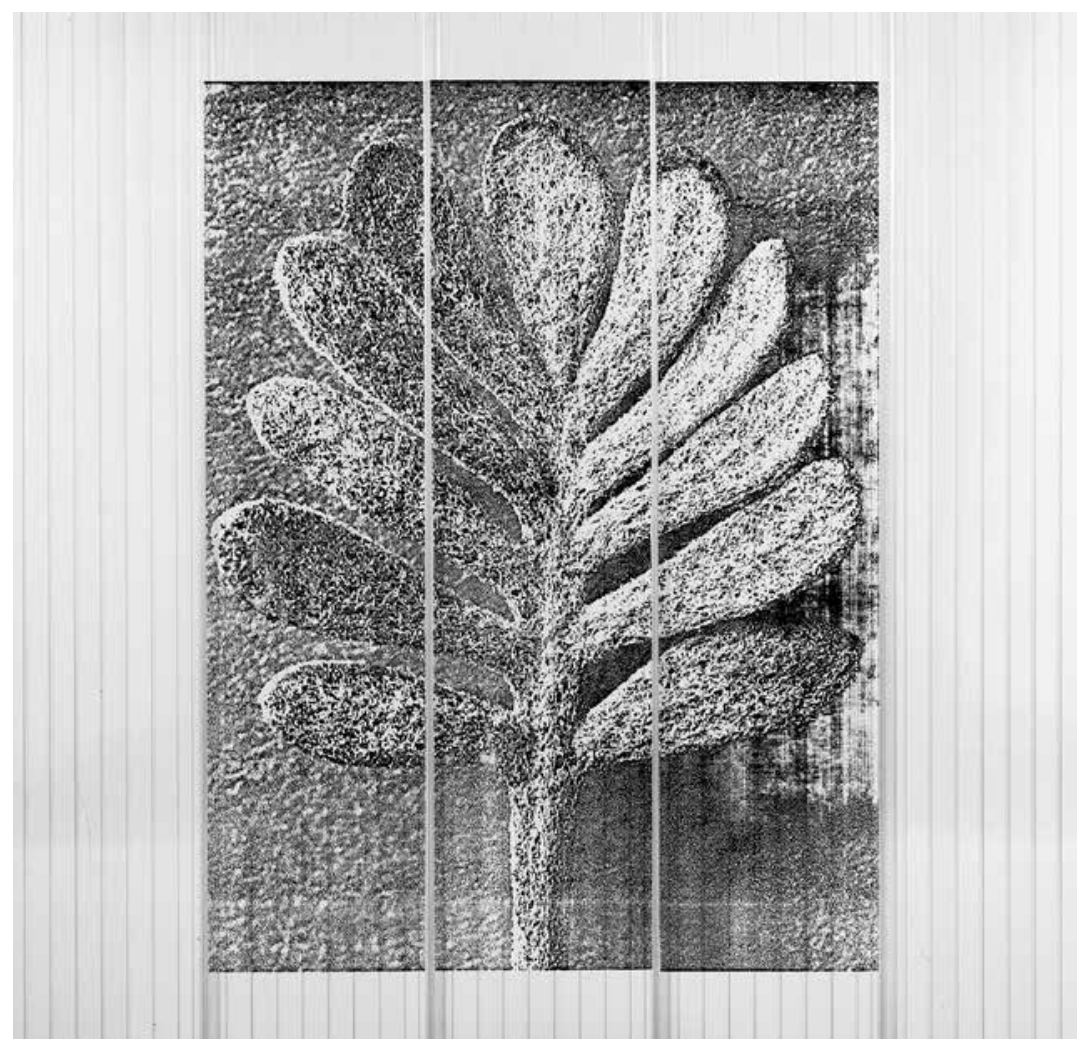

élémentaires, dont les huttes primitives caribéennes offrent l'exemple, dessinent le point de départ de ce qui deviendra la première histoire globale, non de l'art mais de la culture matérielle de l'humanité. Comme chez Bötticher, en revanche, l'ornement est au centre du projet.

2. Herzog \& de Meuron (Bâle), d'après Karl Blossfeldt (Achillea Umbellata, photographie, 1928), panneau de façade de l'usine Ricola Europe (MulhouseBrunnstatt, France), sérigraphie sur acrylique, cadre en aluminium, 1992-1993, New York, Museum of Modern Art, inv. 412.1996.

La lecture de l'ouvrage de Labrusse, parfois difficile, est néanmoins très gratifiante : il apporte au public francophone une riche palette de connaissances et d'éclairages nouveaux. Il mérite d'être traduit en anglais urgemment, ne serait-ce que parce que cette tentative d'une histoire intellectuelle synthétique des études consacrées à l'ornement au XIX ${ }^{e}$ siècle n'a pas d'équivalent.

\section{Vers une histoire globale de l'ornement de surface}

Là où Labrusse trace le tableau de ce qui fut le remplacement du paradigme rhétorique et vitruvien par des modèles linguistique et anthropologique, le volume Histories of Ornament. From Global to Local, publié sous la direction de Gülru Necipoğlu et Alina Payne, interroge le paradigme moderniste eu égard, précisément, à la question de l'ornement, en s'inspirant de la résurgence de l'ornement de surface dans les travaux d'architectes contemporains comme Herzog 8 de Meuron (fig. 2) ou Farshid Moussavi. Dans leur introduction, les directrices de la publication soulignent que la renaissance de l'ornement 
dans la théorie du design aussi bien que de l'architecture ou, au-delà, dans l'histoire de l'art, est aussi saisissante que complexe. L'emploi de l'ornement de surface dans l'architecture contemporaine se démarque de la conception occidentale traditionnelle, venue des ordres, ou conçue comme une représentation de fonctions ou de matériaux. L'ornement de surface peut jouer sur la texture, suggérer par exemple un textile, des surfaces végétales ou la peau elle-même ; il peut utiliser les façades comme un écran de projection pour des photographies, ce qu'ont fait Herzog $\&$ de Meuron sur celles de la bibliothèque de l'École technique d'Eberswalde, réalisée en 1994-1999. Ces ouvrages s'écartent délibérément, dans leur conception, des contextes locaux et historiques, mais dans le même temps, ces façades ornées tirent leur pouvoir suggestif de traditions, comme celle des motifs décoratifs dérivés de végétaux, qui y trouvent une résonance. Si le phénomène récent de l'ornement de surface en architecture est global, il possède néanmoins des racines et produit des résonances locales. À l'origine de ce regain d'intérêt, les auteurs identifient un ensemble assez varié de phénomènes : l'attention nouvelle portée par l'histoire de l'art à la miniature, aux arts décoratifs, en particulier au textile, et au cabinet de curiosités ; mais aussi la réception du livre d'Alfred Gell L'Art et ses agents $^{12}$, publié en 1998, qui a marqué un tournant, ajoutant non seulement l'agency au nombre des enjeux de l'histoire de l'art, mais ouvrant aussi de nouvelles voies pour définir l'ornement de surface, dont les dessins parfois très complexes fournissent à Gell l'un de ses principaux exemples d'artefacts utilisés comme des "pièges à humains " (man traps $)^{13}$. D'un point de vue historique, cet intérêt eut pour précurseurs les rencontres entre histoire de l'art et anthropologie dans les travaux de Semper, Klemm ou Riegl, qui considéraient l'ornement, chacun à leur manière, comme un élément tellement important dans une culture qu'il pouvait constituer un principe de classification stylistique.

L'ambition de ce livre est de créer un nouveau cadre théorique pour l'ornement en architecture, qui vaille partout, du Moyen Âge à nos jours. Il

3. Détail de la façade donnant sur la cour, Florence, Palazzo Spinelli, vers 1460-1470.

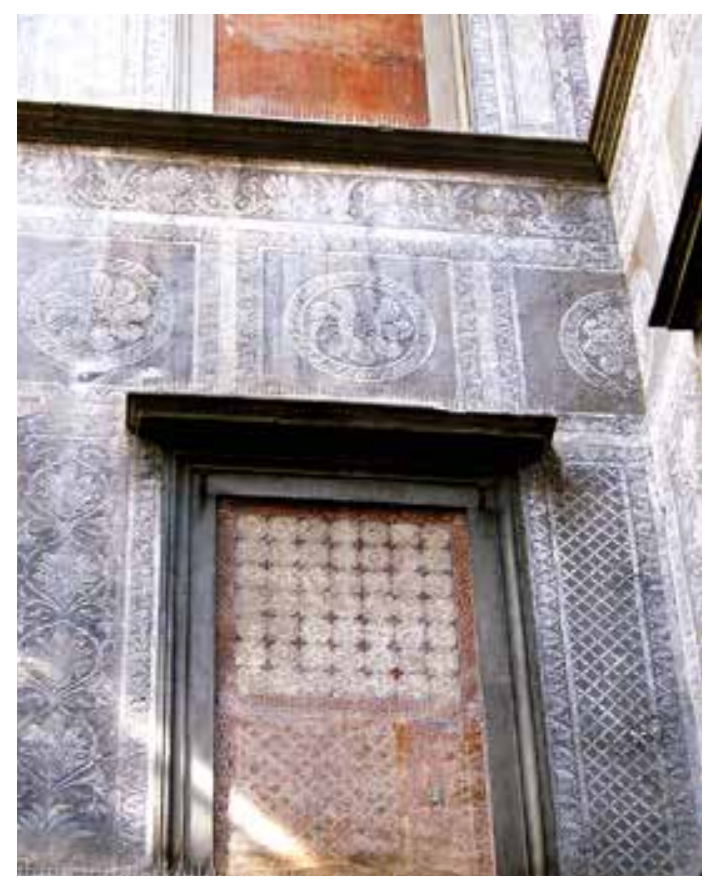
s'attache principalement aux relations entre la portabilité et la diffusion des modèles et des formes, dans le temps et d'une région du monde à une autre. Les auteurs se penchent donc à leur tour sur les problèmes qui avaient retenu l'attention d'un Semper ou d'un Riegl et continuent de préoccuper les archéologues et les anthropologues d'aujourd'hui : comment rendre compte, notamment lorsque fait défaut une documentation extérieure ou textuelle, de l'extension et des résurgences intermittentes de motifs comme l'arabesque ou le grotesque à travers des distances spatiales ou temporelles parfois considérables. Les articles offrent dans leur ensemble une palette incroyablement riche d'études de cas menées dans le monde entier, avec une attention spéciale portée à l'art islamique. L'article de Finbarr Barry Flood sur les motifs des tapis safavides, par exemple, revient sur la tentative d'explication téléologique d'Alois Riegl lorsque celui-ci envisage, dans ses Questions de style ${ }^{14}$, la longue durée ${ }^{15}$ des motifs représentés sur les tapis à l'aune d'un vouloir artistique (Kunstwollen). Le concept échoua 
à éclaircir la question des interruptions et des disjonctions du développement de ces motifs - il était par ailleurs alourdi, pourrait-on ajouter, d'un ensemble d'hypothèses hasardeuses sur la nature de l'ornement et sur l'évolution du naturalisme vers l'abstraction. Riegl ne put résoudre l'hybridité formelle de ces tapis qui offrent au regard des ornements à la fois naturalistes et plus abstraits. Flood propose au contraire ce que l'on pourrait appeler une poétique de la continuité disjonctive, une histoire de l'ornement inspirée par des figures du discours et par des métaphores qui, toutes, nomment la discontinuité : catachrèse, parataxe, et juxtaposition forment les éléments mêmes de sa recherche. Mais ce renoncement à l'ambition d'écrire une continuité narrative qui privilégie la cohésion suggère peut-être, involontairement, le besoin d'un cadre théorique plus large, qui expliquerait comment et pourquoi ces motifs voyagent et changent. Ces cadres plus vastes semblent posés par des études sur la longue durée des styles, notamment celle d'Ann Gunter, dont les récents travaux sur l'art grec et le Moyen-Orient reconstruisent les réseaux commerciaux ou les modèles de diffusion des matériaux et des techniques ${ }^{16}$.

L'article d'Alina Payne sur la technique du sgraffito employée pour la décoration des façades à la Renaissance (fig. 3) offre un nouvel exemple de réexamen, particulièrement stimulant, d'un sujet traité au XIX siècle par Semper, tombé en désuétude, faute de convenir aux types d'ornements sculptés promus par Serlio ou Palladio. Semper y voyait un avatar tardif du tatouage, c'est-à-dire de l'une des premières manifestations du besoin universel qu'a l'humanité de créer des ornements de surface. Payne emprunte une voie différente, en l'occurrence celle du rapprochement avec des techniques similaires au sgraffito, utilisées dans la poterie, la gravure ou le tissage de la soie et du brocard. Elle montre aussi comment cet ornement de façade brouille la distinction entre architectures stables et objets mouvants et amovibles, entre pierre et vêtement, entre intérieur et extérieur. Son article est l'exemple même de ce que signifie repenser l'ornement de surface, à quoi s'attache le livre entier, qui présente dans une série de textes, on peut le dire, de nombreuses facettes du phénomène. Par son étude du sgraffito, Payne nous incite à rompre tant avec les distinctions rhétoriques et vitruviennes qu'avec les oppositions entre fonction et décoration, ou entre forme et contenu ; ou encore avec l'ambition moderniste de présenter l'architecture comme l'art fondateur, témoignant au contraire que l'ornement de surface ne peut être compris qu'en le rapprochant des autres arts et techniques.

Tandis que les études de Guest et Labrusse mettent en lumière la quête de théories fondatrices qui marqua les débuts de la période moderne et le XIX $\mathrm{X}^{\mathrm{e}}$ siècle, lorsque les théoriciens de l'ornement regardaient hors de leur discipline, vers la rhétorique, la théologie, la linguistique, l'anthropologie ou vers d'autres domaines du savoir, plus ou moins stables et définis, Necipoğlu et Payne apportent des pièces qui permettent de penser l'ornement d'une manière radicalement nouvelle, en rupture tant avec ces traditions qu'avec les ambivalences modernistes. Ce qu'elles proposent est une histoire globale, soucieuse de portabilité et de disjonction conjonctive. Dans la généalogie de leur projet, elles citent Gell et des anthropologues de l'art de la fin du XIX siècle. Leur ouvrage, avec l'étendue et la richesse de ses exemples, offre à la recherche de nombreuses pistes, mais il plaide aussi pour une ouverture des études sur l'ornement dans l'histoire de l'art et de l'architecture aux travaux récents sur la diffusion des styles et des techniques s'inspirant du tournant matériel (material turn), de l'attention nouvelle que portent aux matérialités l'archéologie et l'anthropologie.

Cette contribution a été traduite de l'anglais par François Boisivon. 


\section{Caroline van Eck}

Caroline van Eck est professeure d'histoire de l'art à Cambridge. En 2016, elle a été invitée à donner les Slade Lectures à Oxford sur les candélabres Piranèse ; elle a été chercheuse invitée à I'INHA en 2013 et à l'École du Louvre en 2014. Parmi ses récentes publications, on peut citer l'article "Ornament in Europe: From Antiquity to the Twentieth Century " dans I'Oxford Bibliographies in Art History en ligne, et des articles sur Semper dans Gradhiva et la Revue germanique internationale.

\section{NOTES}

1. L'exposition récente consacrée par le Rijksmuseum au style " auriculaire » ou en néerlandais kwabstijl, et à ses migrations de l'argenterie aux stucs ou aux façades de la République néerlandaise du XVII siècle, rend compte de la complexité inhérente à son objet, mais elle constitue, plutôt, une exception ; voir le catalogue par Reinier Baarsen et Ine Castelijn van Beek (dir.), Kwab: Ornament as Art in the Age of Rembrandt, cat. exp. (Amsterdam, Rijksmuseum, 2018), Amsterdam, Rijksmuseum, 2018.

2. Friedrich August Krubsacius, Gedanken von dem Ursprunge, Wachsthum und Verfalle der Verzierungen in den schönen Künsten, Leipzig, Breitkopf, 1759.

3. Caroline van Eck, Classical Rhetoric and the Arts in Early Modern Europe, Cambridge / New York, Cambridge University Press, 2007, p. 13-29.

4. David Summers, Michelangelo and the Language of Art, Princeton, Princeton University Press, 1981, p. 76-77.

5. Pierre Gros, "La Notion d'ornamentum, de Vitruve à Alberti ", dans Perspective, no 1, 2010, p. 130-136 [en ligne, DOI : https://doi.org/10.4000/perspective.1226].

6. Ernst Gombrich, "Style », dans David L. Hills (dir.), International Encyclopedia of the Social Sciences, New York, MacMillan, 1968.

7. Caroline van Eck, «Cannibalisme, tatouage et revêtement : de l'histoire de l'architecture à l'anthropologie de l'art ", dans Gradhiva. Revue de l'anthropologie et des arts du Musée du Quai Branly, n² 25, 2017, p. 26-48.

8. Owen Jones, The Grammar of Ornament, Londres, Day and Son, 1856.

9. Gottfried Semper, Wissenschaft, Industrie und Kunst: Vorschläge zur Anregung nationalen Kunstgefühles bei dem Schlusse der Londoner Industrie-Ausstellung, Braunschweig, F. Vieweg und Sohn, 1852.

10. Gottfried Semper, Du style et de l'architecture : écrits, 1834-1869, Jacques Soulillou (éd.), Marseille, Parenthèses, 2007 ; Style in the Technical and Tectonic Arts, or Practical Aesthetics, Harry Francis Mallgrave (éd.), Los Angeles, Getty Research Institute, 2004 [éd. orig. : Der Stil in den technischen und tektonischen Künsten, oder praktische Aesthetik, ein Handbuch für Techniker, Künstler und Kunstfreunde, Francfort, Verlag für Kunst und Wissenschaft, 1860-1863].
11. « [L]autlos und starr, verräth sich Gedanke und Begriff nur durch characktervolle Zeichen », Carl Bötticher, Die Tektonik der Hellenen, Berlin, Weidmannsche Buchhandlung, 1844-1852, p. XV, notre traduction.

12. Alfred Gell, L'Art et ses agents : une théorie anthropologique, Sophie et Olivier Renaut (trad. fra.), Dijon, Les Presses du réel, 2009 [éd. orig. : Art and Agency: an Anthropological Theory, Oxford (GB), Clarendon Press, 1998].

13. Alfred Gell, "Traps as Artworks and Artworks as Traps ", dans Journal of Material Culture, n 1/1, 1996, p. $15-38$.

14. Alois Riegl, Questions de style. Fondements d'une histoire de I'ornementation, Henri-Alexis Baatsch et Françoise Rolland (trad. fra.), Hubert Damish (préf.), Paris, Hazan, 1992 [éd. orig. : Stilfragen. Grundlegungen zu einer Geschichte der Ornamentik, Berlin, Siemens, 1893].

15. En français dans le texte, $N d T$.

16. Ann Gunter, Greek Art and the Orient, Cambridge / New York, Cambridge University Press, 2009. 